Service social

\title{
Étude descriptive des interventions individuelles et spontanées auprès des enfants et des adolescents exposés à la violence conjugale
}

\author{
Daniel Turcotte, Ginette Beaudoin et Ann Pâquet-Deehy
}

Volume 47, numéro 3-4, 1998

Enfance : enjeux et réalités 2000

URI : https://id.erudit.org/iderudit/706800ar

DOI : https://doi.org/10.7202/706800ar

Aller au sommaire du numéro

Éditeur(s)

École de service social de l'Université Laval

ISSN

1708-1734 (numérique)

Découvrir la revue

Citer cet article

Turcotte, D., Beaudoin, G. \& Pâquet-Deehy, A. (1998). Étude descriptive des interventions individuelles et spontanées auprès des enfants et des adolescents exposés à la violence conjugale. Service social, 47(3-4), 189-220.

https://doi.org/10.7202/706800ar
Résumé de l'article

Cet article présente les résultats d'une recherche sur les pratiques d'intervention auprès des enfants exposés à la violence conjugale. Les données ont été recueillies dans 146 établissements et organismes (CLSC, centres jeunesse, maisons d'hébergement) oeuvrant dans le domaine de la violence. La plupart des interventions sont basées sur l'aide individuelle; elles se distinguent selon trois types : les pratiques féministes formelles, les pratiques féministes ponctuelles et les pratiques éclectiques/systémiques. Des interventions spontanées ont également été relevées dans desmaisons d'hébergement. Le portrait des pratiques d'intervention tracé soulève des questions sur la difficulté pour les intervenants d'évaluer les résultats de leur action, sur le peu de rayonnement des interventions auprès des adolescents et sur la place faite aux parents. 


\title{
Étude descriptive des interventions individuelles et spontanées auprès des enfants et des adolescents exposés à la violence conjugale ${ }^{1}$
}

\author{
Daniel TURCOTTE \\ Ginette BEAUDOIN \\ Ann PÂQUET-DEEHY
}

Cet article présente les résultats d'une recherche sur les pratiques d'intervention auprès des enfants exposés à la violence conjugale. Les données ont été recueillies dans 146 établissements et organismes (CLSC, centres jeunesse, maisons d'hébergement) œuvrant dans le domaine de la violence. La plupart des interventions sont basées sur l'aide individuelle; elles se distinguent selon trois types: les pratiques féministes formelles, les pratiques féministes ponctuelles et les pratiques éclectiques/systémiques. Des interventions spontanées ont également été relevées dans des maisons d'hébergement. Le portrait des pratiques d'intervention tracé soulève des questions sur la difficulté pour les intervenants d'évaluer les résultats de leur action, sur le peu de rayonnement des interventions auprès des adolescents et sur la place faite aux parents.

Research suggested that exposure to woman battering negatively affects the behaviour and emotional well-being of children. The growing awareness of these effects have led to a variety of services for children. But the nature of these services is not well known. This article reports the results of a survey aimed at describing the goals, processes and results of child counselling delivered on a one-to-one basis in community services, child welfare agencies and women's shelters. Informal programs for children in women's shelters are also described.

1 Cette recherche a été financée par le Conseil québécois de la recherche sociale (CQRS). La référence du rapport final est : D. TURCOTTE, G. BEAUDOIN et A. PÂQUETDEEHY (1999), Les pratiques d'intervention auprès des enfants et des adolescents exposés à la violence conjugale, Université Laval, Centre de recherche interdisciplinaire sur la violence intrafamiliale et la violence faite aux femmes (CRI-VIFF).

Service social, vol. 47, nos 3 et $4,1998-1999$ 
La problématique des enfants exposés à la violence conjugale est un phénomène important tant par son incidence que par l'ampleur de ses conséquences. Les recherches menées depuis une dizaine d'années mettent en évidence le fait que ce phénomène a des conséquences négatives marquées sur les enfants (Fantuzzo et Lindquist, 1989; Kolbo, Blakely et Engleman, 1996). En ce sens, Peled (1993) estime que ces enfants doivent devenir un point d'attention majeur dans les prochaines années. Plusieurs indices laissent croire que ce mouvement est déjà amorcé. En effet, plusieurs maisons d'hébergement pour femmes victimes de violence ont mis en place des services d'animation et de thérapie pour les enfants exposés à la violence conjugale (McLeod, 1987; Gaboury et Guénette, 1999). D'autres organismes tels les CLSC et les centres jeunesse ont également fait des actions en ce sens (Beaudoin, Côté, Delisle, Gaboury, Guénette et Lessard, 1998; Pâquet-Deehy, Proulx, Hamel et Chouéri, 1997; Marengère, Champagne et Poirier, 1998).

C'est avec la préoccupation de cerner de façon plus précise les développements actuels dans ce domaine que fut réalisée cette recherche dont les trois objectifs sont :

- relever les pratiques d'intervention s'adressant aux enfants et aux adolescents exposés à la violence conjugale qui sont réalisées, exclusivement ou en partenariat, par les établissements du réseau de la santé et des services sociaux et par les maisons d'hébergement pour femmes victimes de violence;

- décrire les principales caractéristiques de ces pratiques et en élaborer une typologie;

- relever les éléments critiques associés à leur mise en application de façon à dégager des pistes pour le développement et l'amélioration de ces pratiques.

Le présent texte présente un portrait des pratiques d'intervention qui ont été recensées dans 146 établissements et organismes œuvrant dans le domaine de la violence conjugale au Québec. Après une brève présentation de la problématique des enfants et adolescents exposés à la violence conjugale, le texte fait état, dans une deuxième partie, de la méthode utilisée pour conduire la 
recherche. En troisième partie, les données sur les interventions sont présentées en distinguant les pratiques d'intervention basées sur l'aide individuelle et les interventions spontanées. La conclusion esquisse quelques enjeux actuels de l'intervention auprès des enfants et des adolescents exposés à la violence conjugale.

\section{LA RÉALITÉ DES ENFANTS EXPOSÉS À LA VIOLENCE CONJUGALE}

L'exposition à la violence conjugale couvre une vaste gamme d'expériences, notamment assister à des scènes de violence entre les parents, entendre des éclats de voix, voir les marques des coups portés, percevoir la détresse dans le regard du parent, etc. (Jaffe, Wolfe et Wilson, 1990). Ainsi, les enfants peuvent tout aussi bien observer directement les comportements de violence qu'en constater les conséquences après coup (Jaffe et collab., 1990; Peled et Davis, 1995).

Pour comprendre la réalité des enfants qui vivent dans un contexte de violence conjugale, il faut aussi être conscient de certaines de ses caractéristiques, notamment du cycle de la violence (tension, agression, déresponsabilisation, rémission et réconciliation). Non seulement les enfants sont témoins de la violence, mais ils vivent dans un climat de tension, de friction et de peur, qui éclate, diminue et se reconstruit. À cet égard, leur vécu se rapproche de celui de leur mère, tout en s'en distinguant. Leurs émotions peuvent aller de la peur à la terreur et de l'anxiété à l'insécurité, jusqu'à des sentiments plus positifs durant les périodes plus calmes.

Tout comme c'est le cas pour la violence familiale et la violence conjugale, l'ampleur réelle du nombre d'enfants exposés à la violence conjugale demeure une réalité mal connue; des chercheurs estiment à 2,6 millions le nombre de Canadiennes qui ont été violentées en contexte conjugal. Quatre fois sur dix (39\%), ces femmes ont rapporté que leurs enfants avaient été témoins de cette violence, laquelle se présentait le plus souvent sous forme de violence physique (61\%). En fait, si les familles au sein desquelles il y a présence de violence conjugale comptent en moyenne un enfant, il est possible d'affirmer que plus d'un million d'enfants, dont plusieurs sont maintenant adultes, ont vu leur mère être violentée (Johnson, 1996). Aux États-Unis, les données 
de l'enquête nationale sur la violence familiale suggèrent qu'il y a, chaque année, 10 millions d'enfants exposés à la violence conjugale (Holden, 1998).

\section{LES CONSÉQUENCES DE LA VIOLENCE CONJUGALE SUR LES ENFANTS}

Les résultats de recherches ont conduit à distinguer cinq catégories de difficultés ou de symptômes que peuvent éprouver ces enfants: 1) des problèmes de comportements (externalizing behavior problems or conduct), 2) des problèmes émotionnels (internalizing behavior problems), 3) des problèmes de fonctionnement social, 4) des problèmes d'ordre cognitif et scolaire et 5) des problèmes physiques (Fantuzzo et Lindquist, 1989; Kolbo, Blakely et Engleman, 1996). Le tableau 1 présente une synthèse des difficultés décelées chez ces enfants.

Jaffe et ses collègues (1990) et, plus récemment, Hughes (1997) ainsi que Wolak et Finkelhor (1998) distinguent entre les effets directs et les effets indirects que peut avoir la violence conjugale chez les enfants et les adolescents qui y sont exposés. Les effets directs comprennent le danger physique pour l'enfant, les problèmes émotionnels et comportementaux et l'apprentissage de comportements agressifs. Les effets indirects concernent la santé physique et mentale déficiente de la mère résultant du stress d'avoir été violentée, ce qui peut dans certaines situations influencer sa capacité à jouer son rôle parental et l'attachement mèreenfant.

L'exposition à la violence conjugale peut également avoir des conséquences à long terme. Ainsi, Carlson (1990) mentionne que même si certains enfants apparaissent ne pas être affectés à court terme, des effets peuvent se manifester plus tard, notamment dans leurs relations interpersonnelles et leurs rapports amoureux. Par exemple, une femme qui vit avec un homme dont le père était violent avec sa conjointe est trois fois plus susceptible d'être agressée par son propre conjoint que celle qui vit avec un homme dont le père n'était pas violent envers sa conjointe (Johnson, 1996). L'étude de Hennings, Leintenberg, Coffey, Turner et Bennett (1996) indique que les femmes qui ont été exposées à la violence conjugale durant leur enfance démontrent un niveau plus élevé de détresse psychologique et un niveau 
moins élevé d'ajustement social. Pour leur part, les conjoints violents sont plus susceptibles d'avoir été témoins ou victimes de violence dans leur famille d'origine (Straus, Gelles et Steinmetz, 1980; Rosenbaum et O'Leary, 1981). L'estimation de la proportion d'hommes abuseurs qui proviennent de familles possédant une histoire de violence oscille entre 50 \% (Sonkin, Martin et Walker, 1985 ) et $80 \%$ (Roy, 1982) selon les études. Selon Wolfe (1999), le meilleur prédicteur de la violence conjugale est l'exposition à des actes de violence dans la famille d'origine.

En résumé, bien que les données disponibles ne présentent qu'une vue partielle du phénomène des enfants exposés à la violence conjugale, ils permettent d'affirmer qu'il s'agit là d'une réalité répandue qui fait souffrir les enfants et qui se révèle rarement sans conséquences, bien que certaines ne soient perceptibles qu'à long terme. Cette problématique est d'autant plus préoccupante qu'elle se présente généralement en cooccurrence avec d'autres problèmes (Hughes et collab., 1989; Chénard et collab., 1990; Jaffe, Wolfe et Wilson, 1990; Petchers, 1995; Rinfret-Raynor, Pâquet-Deehy, Larouche et Cantin, 1992; McKay, 1994). 


\section{Tableau 1 \\ Synthèse des difficultés des enfants exposés à la violence conjugale}

\begin{tabular}{|c|c|}
\hline $\begin{array}{l}\text { Problèmes de } \\
\text { comportements }\end{array}$ & $\begin{array}{l}\text { - Extériorisation des émotions se traduisant } \\
\text { entre autres par des comportements agressifs } \\
\text { et des actes délinquants } \\
\text { (Hughes et Barad, 1983; Forstron-Cohen et } \\
\text { Rosenbaum, 1985; Wolfe, Jaffe, Wilson et Zak, } \\
\text { 1985; Jaffe, Wolfe, Wilson et Zak, 1986; } \\
\text { Hughes, Parkinson et Vargo, 1989; Hughes et } \\
\text { collab., 1989; Carlson, 1990) }\end{array}$ \\
\hline Problèmes émotionnels & $\begin{array}{l}\text { - Faible estime de soi } \\
\text { - } \text { Anxiété, dépression, irritabilité } \\
\text { - } \text { Idéations suicidaires, phobies, insomnies } \\
\text { - } \text { Réactions analogues au stress post- } \\
\text { traumatique } \\
\text { (Fantuzzo et Lindquist, 1989; Wolfe et collab., } \\
\text { 1985; Hughes, 1988; Fantuzzo, 1991; } \\
\text { Chénard, 1994) }\end{array}$ \\
\hline $\begin{array}{l}\text { Difficultés sur le plan du } \\
\text { fonctionnement social }\end{array}$ & $\begin{array}{l}\text { - Déficit des habiletés sociales } \\
\text { - } \text { Rejet par les pairs } \\
\text { - Stratégies violentes de résolution de conflits } \\
\text { - } \text { Faible empathie } \\
\text { (Jaffe, Wolfe, Wilson et Zak, 1985; Wolfe et } \\
\text { collab., 1985, Rudo, Powell et Dunlap, 1998). }\end{array}$ \\
\hline $\begin{array}{l}\text { Problèmes d'ordre cognitif } \\
\text { et scolaire }\end{array}$ & $\begin{array}{l}\text { - Déficience des habiletés verbales et } \\
\text { intellectuelles } \\
\text { - Retard scolaire } \\
\text { (Westra et Martin, 1981; Chénard et collab., } \\
\text { 1990; Chénard, 1994) }\end{array}$ \\
\hline $\begin{array}{l}\text { Problèmes de santé } \\
\text { physique }\end{array}$ & $\begin{array}{l}\text { - Allergies, affections cutanées, maux de tête, } \\
\text { asthme } \\
\text { (Kérouak et collab., 1986; Chénard et collab., } \\
\text { 1990; Chénard, 1994; Arroyo et Eth, 1995; } \\
\text { Silvern, Karyl et Landis, 1995; Kilpatrick et } \\
\text { Williams, 1997; Glodich, 1998) }\end{array}$ \\
\hline
\end{tabular}




\section{LES VOIES D'ACTION}

II est possible de recenser dans les écrits la description de différentes voies d'action auprès des enfants et des adolescents exposés à la violence conjugale. Certaines de ces actions consistent en des interventions individuelles auprès des jeunes (Davis, 1991, Burman et Allen Meares, 1994; Silvern et Landis, 1995; Arroyo et Eth, 1995). D'autres s'intègrent à des programmes plus vastes dans lesquels sont offerts un éventail de services individuels ou de groupe à l'intention des mères, des enfants ou des adolescents et, dans certains cas, des conjoints violents; c'est le cas, notamment, dans le programme expérimenté dans les Centres de la jeunesse et de la famille Batshaw (Pâquet-Deehy, Proulx et Hamel, 1997) et dans le "Domestic Abuse Project " (Grunski et collab., 1988; Johnson et Montgomery, 1990; Peled et Edleson, 1992; Peled et Davis, 1995 et Peled et Edleson, 1995). D'autres, enfin, se greffent aux actions mises en place dans les maisons d'hébergement pour femmes victimes de violence (Hughes, 1982;

Alessi et Hearn, 1984; Jaffe, Wilson et Wolfe, 1986; Gibson et Gutierrez, 1991). Ces dernières interventions s'intègrent, le plus souvent, dans un ensemble de services offerts en parallèle aux enfants et à leur mère.

Le portrait que présentent les revues scientifiques et professionnelles renvoie cependant le plus souvent à des expériences qui sont menées chez nos voisins du Sud. De sorte que la réalité québécoise en matière d'intervention auprès des enfants et des adolescents exposés à la violence conjugale demeure méconnue. C'est pour corriger cette situation que la présente recherche a été réalisée.

\section{LA MÉTHODE DE RECHERCHE}

Trois types d'organismes ont été ciblés comme sources d'information : les centres jeunesse, les CLSC et les maisons d'hébergement pour femmes victimes de violence conjugale. Ces ressources ont été privilégiées parce qu'elles sont les plus susceptibles d'avoir développé des pratiques d'intervention auprès des enfants et des adolescents exposés à la violence conjugale. 
Les pratiques qui ont été retenues devaient répondre aux critères suivants: (1) avoir été réalisées au cours des 12 derniers mois, (2) avoir des objectifs d'intervention qui sont explicitement liés à la réalité des enfants ou des adolescents exposés à la violence conjugale et (3) avoir donné lieu à des interventions directes auprès d'enfants ou d'adolescents. Ces pratiques pouvaient couvrir à la fois des programmes structurés, des interventions planifiées ou des interventions spontanées et elles pouvaient avoir été réalisées tout autant dans le cadre d'interventions individuelles qu'en contexte de groupe.

\section{LA COLLECTE DES DONNÉES}

Deux modalités de collecte des données ont été utilisées : l'entrevue téléphonique et le questionnaire auto-administré. L'entrevue téléphonique a été la modalité privilégiée le plus souvent. Lors d'un premier contact téléphonique, l'intervieweur convenait avec l'informateur clé du moment le plus approprié pour la réalisation de l'entrevue. Une copie du questionnaire était alors envoyée au répondant par courrier ou par télécopieur afin que celui-ci puisse se préparer à l'entrevue. Au moment convenu, l'intervieweur communiquait à nouveau avec le répondant afin de recueillir l'information sur ses pratiques d'intervention; les réponses étaient alors inscrites sur le questionnaire, permettant ainsi de respecter les termes et expressions utilisés par l'informateur.

Trois questionnaires construits par l'équipe de recherche ont été utilisés pour la collecte des données selon que les pratiques d'intervention étudiées s'appuyaient sur l'aide individuelle, l'intervention de groupe ou l'action spontanée. Les questionnaires sur les interventions individuelles et de groupe comportent des questions ouvertes et des questions fermées dont plusieurs sont communes; le questionnaire sur les actions spontanées comprend exclusivement des questions ouvertes.

\section{LA NATURE DES INFORMATIONS RECUEILLIES}

Les éléments abordés dans les questionnaires ont été déterminés à la suite d'une revue des écrits sur les pratiques d'intervention auprès des enfants exposés à la violence conjugale (Jaffe et collab., 1990; Peled et Davis, 1995) et sur certains programmes 
de prévention (Morris et Fitz-Gibbon, 1978; Mercier, 1985; Belsky, 1984; Powell, 1988; Weiss, 1988; Whitebook et collab., 1989; MSSS, 1991; Blanchet et collab., 1993; Guberman et collab., 1993). Les éléments suivants ont été retenus pour encadrer l'analyse des pratiques d'intervention auprès des enfants et des adolescents exposés à la violence conjugale : les caractéristiques des intervenants, le contexte organisationnel, les liens avec la communauté, la nature des interventions (clientèle cible, objectifs, modalités, activités) et les résultats.

\section{LE PROFIL DES RÉPONDANTS}

Cette stratégie de collecte des données a permis d'obtenir de l'information auprès d'intervenants et d'intervenantes de 146 établissements et organismes sur les 264 ciblés au départ, soit $55,3 \%$. Comme l'indique le tableau 2, le taux de réponse le plus élevé se situe chez les maisons d'hébergement (72,9\%), alors que le taux le plus faible se retrouve chez les CLSC.

\section{Tableau 2}

Taux de réponse selon le cadre organisationnel

\begin{tabular}{|l|c|c|c|c|}
\hline $\begin{array}{l}\text { Nombre d'organismes ayant } \\
\text { répondu }\end{array}$ & CLSC & $\begin{array}{c}\text { Centres } \\
\text { jeunesse }\end{array}$ & $\begin{array}{c}\text { Maisons } \\
\text { d'hébergement }\end{array}$ & TOTAL \\
\hline - Nombre d'organismes & 67 & 9 & 70 & 146 \\
$\begin{array}{l}\text { participants } \\
\text { - Nombre d'organismes } \\
\text { sollicités }\end{array}$ & 150 & 18 & 96 & 264 \\
- Proportion & $44,6 \%$ & $50,0 \%$ & $72,9 \%$ & $55,3 \%$ \\
\hline
\end{tabular}

Le motif qui explique le plus fréquemment la non-participation à l'étude est l'impossibilité, en dépit d'efforts répétés, d'obtenir le nom d'un répondant ou d'entrer en contact avec l'informateur clé à l'intérieur de la période consacrée à la collecte des données. Dans le cas de plusieurs organismes, cette situation est attribuable au fait que, selon l'information obtenue, leurs pratiques d'intervention auprès d'enfants ou d'adolescents exposés à la violence conjugale ne répondaient pas aux critères de la recherche au moment de la collecte des données. Enfin, quelques organismes ont refusé de participer en raison d'un surplus de travail ou d'un contexte de restructuration organisationnelle. 
L'information obtenue sur les 146 organismes et établissements participants a conduit à documenter 151 pratiques basées sur l'aide individuelle et 65 pratiques de type " action spontanée ».

\section{LES PRATIQUES D'INTERVENTION BASÉES SUR L'AIDE INDIVIDUELLE}

Au total, 151 intervenantes et intervenants (la plupart [84,7\%] des femmes) ont fourni une description de leurs pratiques d'intervention basées sur l'aide individuelle : 86 d'entre eux œuvraient en CLSC, 13 dans un centre jeunesse et 52 en maisons d'hébergement. Pour près de la moitié (44,9\%), l'approche féministe représente la principale assise théorique de leur intervention; cette situation vaut tout autant pour les répondants des maisons d'hébergement $(70 \%)$, des CLSC $(30,9 \%)$ et des centres jeunesse $(38,5 \%)$. Ce sont ensuite les approches éclectique $(17,7 \%)$, systémique $(9,5 \%)$ et psycho-éducationnelle $(8,8 \%)$ qui sont le plus fréquemment identifiées comme principale assise théorique.

\section{LES OBJECTIFS DES INTERVENTIONS INDIVIDUELLES}

Les objectifs visés par les interventions individuelles se divisent en deux grandes catégories: les objectifs de processus et les objectifs de résultats (tableau 3 ). Les objectifs de processus correspondent aux moyens qui sont privilégiés pour produire les résultats recherchés. Trois objectifs ont été associés à cette catégorie : (1) permettre aux enfants d'exprimer les émotions provoquées par leur expérience de violence conjugale, objectif qui se retrouve chez près de $60 \%$ des répondantes, (2) répondre aux besoins immédiats des enfants, objectif mentionné par 32,6\% des répondantes et (3) créer une relation positive avec le jeune (16,6\%). Les objectifs de résultats traduisent les effets visés par les interventions mises en place. Ces effets peuvent être immédiats et en lien direct avec la violence, comme apprendre au jeune des scénarios de protection, ou ils peuvent avoir une visée à plus long terme, comme prévenir la violence dans les relations amoureuses. Les objectifs à court terme renvoient aux éléments suivants: atténuer les impacts négatifs causés par le fait d'avoir été exposé à la violence conjugale, faire apprendre des scénarios de protection, amener le jeune à développer une analyse plus objective de la situation de violence familiale, le préparer à vivre les transitions qui vont marquer la vie de la famille et l'aider à faire 
face à la crise actuelle. Les objectifs à moyen terme sont le développement d'une meilleure capacité à gérer les émotions associées à la violence conjugale et la restauration de l'estime de soi. Enfin, les objectifs à plus long terme sont liés à la prévention de la violence et ils s'appuient généralement sur des stratégies comme l'augmentation des connaissances des jeunes sur le phénomène de la violence familiale, la prise de conscience des stéréotypes sexuels et l'établissement de rapports égalitaires entre les garçons et les filles.

Cinq objectifs de résultats sont mentionnés par plus du quart de l'ensemble des répondantes; ce sont: (1) atténuer les impacts négatifs de l'exposition à la violence conjugale, (2) prévenir la violence, (3) développer la capacité de gérer les émotions associées à la violence, (4) restaurer l'estime de soi et (5) faciliter l'apprentissage de scénarios de protection. On observe toutefois certaines différences dans les réponses selon le type d'organisme. Ainsi, dans les centres jeunesse plus de la moitié des répondantes $(61,5 \%)$ ont pour objectif d' " aider l'enfant à apprendre des scénarios de protection $॥$; cette proportion est beaucoup plus faible chez celles qui travaillent en CLSC $(29,4 \%)$ ou en maisons d'hébergement $(11,5 \%)$. Par ailleurs, du côté des maisons d'hébergement, plus de six répondantes sur dix $(65,3 \%)$ indiquent viser la prévention de la violence; cet objectif est beaucoup moins présent dans les CLSC (28,2\%) et dans les centres jeunesse (15,3\%). 
Tableau 3

Objectifs visés selon le type d'établissement*

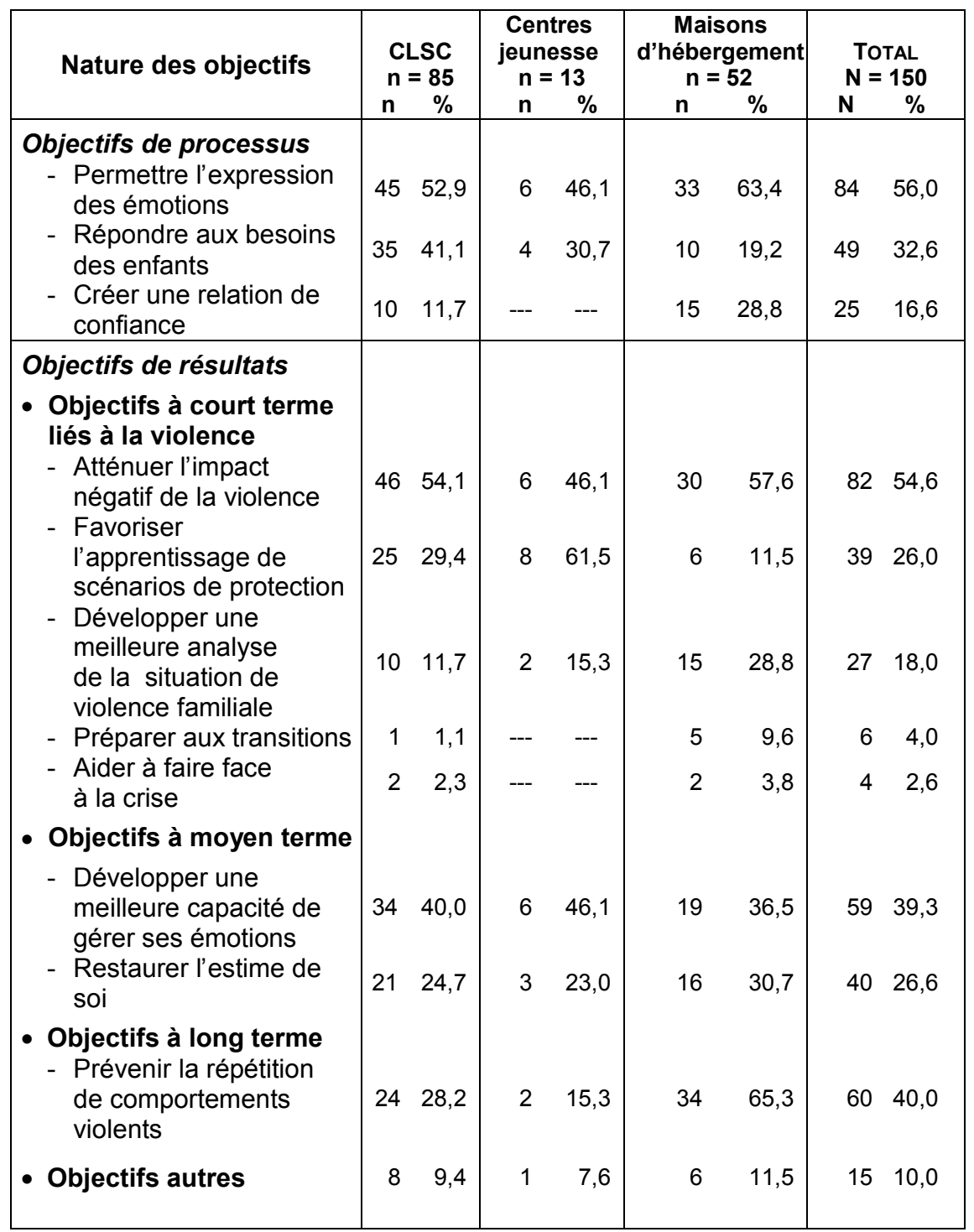

* Les pourcentages sont calculés en fonction du nombre de répondantes; leur total peut être supérieur à 100 car les catégories ne sont pas exclusives. 


\section{LES MODALITÉS D'INTERVENTION}

Que ce soit en CLSC, en centres jeunesse ou en maisons d'hébergement, la majorité des répondantes privilégient un modèle d'intervention à court terme : près du tiers $(31,6 \%)$ réalisent leur plan d'intervention à l'intérieur de cinq rencontres et plus de trois sur quatre $(78,2 \%)$ le font en moins de 11 rencontres. Le nombre moyen de rencontres en CLSC et en maisons d'hébergement se situe respectivement à $8,3( \pm 4,5)$ et $8,0( \pm 5,2)$, alors que chez les répondantes des centres jeunesse la moyenne se chiffre à $13,6( \pm 6,6)$ rencontres.

Tableau 4

Nombre, durée et fréquence des interventions

\begin{tabular}{|c|c|c|c|c|c|c|c|c|}
\hline \multirow{2}{*}{ Nature des objectifs } & \multicolumn{2}{|c|}{ CLSC } & \multicolumn{2}{|c|}{$\begin{array}{c}\text { Centres } \\
\text { jeunesse }\end{array}$} & \multicolumn{2}{|c|}{$\begin{array}{c}\text { Maisons } \\
\text { d'hébergement }\end{array}$} & \multicolumn{2}{|c|}{ Total } \\
\hline & $\mathbf{n}$ & $\%$ & $\mathbf{n}$ & $\%$ & $\mathbf{n}$ & $\%$ & $\mathbf{N}$ & $\%$ \\
\hline Nombre d'interventions & \multicolumn{2}{|c|}{$(n=85)$} & \multicolumn{2}{|c|}{$(n=11)$} & \multicolumn{2}{|c|}{$(\mathrm{n}=37)$} & \multicolumn{2}{|c|}{$(N=133)$} \\
\hline 1 à 5 & 27 & 31,7 & --- & -- & 15 & 40,5 & 42 & 31,6 \\
\hline 6 à 10 & 43 & 50,6 & 6 & 54,5 & 13 & 35,1 & 62 & 46,6 \\
\hline 11 à 15 & 11 & 12,9 & 1 & 9,0 & 6 & 16,2 & 18 & 13,5 \\
\hline 16 et plus & 4 & 4,7 & 4 & 36,4 & 3 & 8,1 & 11 & 8,3 \\
\hline Moyenne (écart-type) & 8,3 & $(4,5)$ & 13,6 & $(6,6)$ & 8,0 & $(5,2)$ & 8,9 & $(6,3)$ \\
\hline Durée des interventions & \multicolumn{2}{|c|}{$(n=86)$} & \multicolumn{2}{|c|}{$(n=12)$} & \multicolumn{2}{|c|}{$(n=47)$} & \multicolumn{2}{|c|}{$(\mathrm{N}=145)$} \\
\hline $1-30$ & 10 & 11,6 & 1 & 8,3 & 19 & 40,4 & 30 & 20,7 \\
\hline $31-59$ & 32 & 37,2 & 3 & 25,0 & 12 & 25,5 & 47 & 32,4 \\
\hline 60 & 32 & 37,2 & 7 & 58,3 & 8 & 17,0 & 47 & 32,4 \\
\hline 61 et plus & & 14,0 & 1 & 8,3 & 8 & 17,0 & 21 & 14,5 \\
\hline Moyenne (écart-type) & 55,6 & $(20,2)$ & 55,8 & $(11,0)$ & 49,1 & $(25,5)$ & 53,5 & $(21,6)$ \\
\hline $\begin{array}{l}\text { Fréquence des } \\
\text { interventions }\end{array}$ & \multicolumn{2}{|c|}{$(n=85)$} & \multicolumn{2}{|c|}{$(n=12)$} & \multicolumn{2}{|c|}{$(n=48)$} & \multicolumn{2}{|c|}{$(\mathrm{N}=145)$} \\
\hline Bihebdomadaire & 5 & 5,9 & -- & --- & 6 & 12,5 & 11 & 7,6 \\
\hline Hebdomadaire & 35 & 41,2 & 4 & 33,3 & 23 & 47,9 & 62 & 42,7 \\
\hline Bimensuelle & 23 & 27,0 & 6 & 50,0 & 4 & 8,3 & 33 & 22,7 \\
\hline Mensuelle & 8 & 9,4 & 2 & 16,6 & --- & --- & 10 & 6,9 \\
\hline Au besoin & 14 & 16,5 & --- & --- & 13 & 27,0 & 27 & 18,6 \\
\hline
\end{tabular}

Au regard de la durée des rencontres, les répondantes des centres jeunesse apparaissent également privilégier des rencontres plus longues; plus de la moitié $(58,3 \%)$ indiquent que leurs entrevues durent au moins une heure. Par ailleurs, en CLSC et en 
maisons d'hébergement, respectivement $37,2 \%$ et $17 \%$ des répondantes privilégient des rencontres ayant une telle durée. En fait, les rencontres sont légèrement plus longues dans les centres jeunesse (55,8 minutes en moyenne) et les CLSC (55,6 minutes) que dans les maisons d'hébergement (49,1 minutes). Par ailleurs, en centres jeunesse les interventions sont surtout bimensuelles, alors qu'en CLSC et en maisons d'hébergement la fréquence la plus répandue est l'intervention hebdomadaire; elle est privilégiée par plus de quatre répondantes sur dix $(41,2 \%$ et $47,9 \%)$.

\section{LA PLACE DES PARENTS}

La place faite aux parents dans la démarche d'intervention varie considérablement d'une répondante à l'autre en ce qui a trait au père; par contre, presque toutes cherchent à impliquer la mère dans leur démarche. Dans les centres jeunesse et les maisons d'hébergement, toutes les répondantes indiquent que " la mère a un rôle dans l'intervention auprès de l'enfant ", tandis que dans les CLSC une forte majorité $(93,0 \%$ ) expriment une position identique.

Les buts visés par l'association de la mère au processus d'intervention sont variés. Dans certains cas, les répondantes veulent ainsi améliorer la situation de la mère : soutenir ou aider la mère à satisfaire ses besoins personnels, l'aider à bâtir des stratégies adéquates de résolution de conflits et l'amener à reprendre du contrôle sur sa vie. Dans d'autres cas, cette association est vue comme un moyen d'assurer une meilleure réponse aux besoins de l'enfant: obtenir la collaboration de la mère pour qu'elle appuie l'intervention, améliorer sa capacité à répondre aux besoins de l'enfant et la sensibiliser aux conséquences de la violence sur l'enfant. Enfin, pour certaines répondantes, elle apparaît comme une façon de répondre à la fois aux besoins de la mère et à ceux de l'enfant : assurer la protection de la mère et de l'enfant, améliorer la relation mère-enfant et diriger la mère et l'enfant vers des ressources en mesure de répondre à leurs besoins.

La contribution du père à la démarche d'intervention se présente comme un aspect plus problématique et la position des répondantes quant à l'intérêt d'associer le père à l'intervention 
varie considérablement. C'est en centres jeunesse que se retrouve l'intérêt le plus marqué en faveur de l'association du conjoint violent à la démarche d'intervention : trois répondantes sur quatre $(76,9 \%)$ indiquent qu'elles tentent d'impliquer le père dans leur intervention; en CLSC, moins de la moitié $(45,3 \%)$ répondent de la même façon. En maisons d'hébergement, elles ne tentent pas d'associer le père à leur action.

Différents buts sont visés par les répondantes lorsqu'elles associent le conjoint violent à leur démarche d'intervention. Les plus répandus sont ${ }^{2}$ : (1) le conscientiser aux besoins de l'enfant (49\%), (2) le responsabiliser face à ses comportements violents $(40,4 \%)$, et (3) l'intégrer dans une démarche d'aide en le dirigeant vers des ressources adaptées à ses besoins (34,7\%).

\section{LES RÉSULTATS DE L'INTERVENTION}

La majorité des répondantes des centres jeunesse $(76,9 \%)$ et des maisons d'hébergement $(64,7 \%)$ indiquent qu'elles évaluent leurs interventions. En CLSC, une tendance inverse est observée; la majorité des répondantes $(64,2 \%)$ donnent une réponse négative. Faut-il en conclure qu'il se fait moins d'évaluation en CLSC que dans les autres types d'organismes ? II est difficile de répondre à cette question, car il se peut que l'écart observé soit attribuable à l'ambiguïté de la question. En effet, comme le terme " évaluation » n'était pas défini, il est possible que certaines répondantes de CLSC l'aient interprété dans un sens plus formel.

\footnotetext{
${ }^{2}$ Ces catégories de réponses ne sont pas exclusives.
} 
Tableau 5

Procédures d'évaluation*

\begin{tabular}{|c|c|c|c|c|c|c|c|c|}
\hline Stratégie d'évaluation & & $\begin{array}{r}\text { SC } \\
=30 \\
\% \\
\end{array}$ & \multicolumn{2}{|c|}{$\begin{array}{c}\text { Centres } \\
\text { jeunesse } \\
n=6\end{array}$} & \multicolumn{2}{|c|}{$\begin{array}{c}\text { Maisons } \\
\text { d'hébergement } \\
n=32\end{array}$} & \multicolumn{2}{|c|}{$\begin{array}{l}\text { Total } \\
N=68 \\
N \quad \%\end{array}$} \\
\hline Bilan avec le client & 19 & 63,3 & 2 & 33,3 & 23 & 71,9 & 44 & 64,7 \\
\hline $\begin{array}{l}\text { Sommaire d'intervention } \\
\text { par l'intervenante }\end{array}$ & 13 & 43,3 & 4 & 66,6 & 4 & 12,5 & 21 & 30,9 \\
\hline $\begin{array}{l}\text { Évaluations formelles } \\
\text { basées sur des procédures } \\
\text { ou des instruments }\end{array}$ & 4 & 13,3 & -- & -- & 4 & 12,5 & 8 & 11,7 \\
\hline Évaluation continue & -- & -- & -- & -- & 1 & 3,1 & 1 & 1,5 \\
\hline $\begin{array}{l}\text { Appréciation de } \\
\text { collaborateurs externes }\end{array}$ & 3 & 10,0 & 1 & 16,6 & 1 & 3,1 & 5 & 7,3 \\
\hline $\begin{array}{l}\text { Autres (notes aux dossiers, } \\
\text { observations, contacts } \\
\text { post-intervention) }\end{array}$ & 4 & 13,3 & 2 & 33,3 & 3 & 9,4 & 9 & 13,2 \\
\hline
\end{tabular}

* Les pourcentages sont calculés en fonction du nombre de répondantes; leur total peut être supérieur à 100 car les catégories ne sont pas exclusives.

Quoi qu'il en soit, lorsque l'intervention fait l'objet d'une évaluation c'est le plus souvent à l'aide de procédures informelles, telles que : 1) le bilan avec les clients (enfants et parents), 2) le sommaire d'intervention et 3) l'appréciation de collaborateurs externes. Les évaluations formelles basées sur des procédures ou sur des instruments standardisés sont peu répandues. Plus spécifiquement, comme l'indique le tableau 5 , chez les répondantes qui évaluent leurs interventions, le bilan avec le client est la pratique la plus courante dans les maisons d'hébergement $(71,9 \%)$ et dans les CLSC (63,3\%); dans les centres jeunesse, l'évaluation consiste le plus souvent en un bilan effectué par l'intervenante seulement.

Bien qu'elles utilisent rarement des procédures formelles d'évaluation, les répondantes n'en ont pas moins une opinion sur la portée de leur action, même si plusieurs mentionnent qu'il leur est difficile de savoir dans quelle mesure leurs objectifs sont véritablement atteints, notamment en ce qui a trait aux objectifs à long terme. En fait, la plupart situent leur action comme une amorce de 
changement; elles soulignent que la durée de leur intervention est généralement trop brève pour qu'elles atteignent tous leurs objectifs.

Tableau 6

Résultats attribués aux interventions*

\begin{tabular}{|c|c|c|c|c|c|c|c|c|}
\hline Effets des interventions & \multicolumn{2}{|c|}{$\begin{array}{l}\text { CLSC } \\
n=54 \\
n \quad \%\end{array}$} & \multicolumn{2}{|c|}{$\begin{array}{l}\text { Centres } \\
\text { jeunesse } \\
n=9\end{array}$} & \multicolumn{2}{|c|}{$\begin{array}{c}\text { Maisons } \\
\text { d'hébergement } \\
n=20\end{array}$} & \multicolumn{2}{|c|}{$\begin{array}{l}\text { Total } \\
\mathbf{N}=\mathbf{8 3}\end{array}$} \\
\hline $\begin{array}{l}\text { Offrent un cadre qui } \\
\text { favorise l'expression des } \\
\text { émotions }\end{array}$ & 24 & 44,4 & 3 & 33,3 & 12 & 60,0 & 39 & 47,0 \\
\hline $\begin{array}{l}\text { Contribuent à réduire les } \\
\text { impacts négatifs de } \\
\text { l'expérience de la violence }\end{array}$ & 16 & 29,6 & 3 & 33,3 & 9 & 45,0 & 28 & 33,7 \\
\hline $\begin{array}{l}\text { Conduisent à l'acquisition } \\
\text { de stratégies non violentes } \\
\text { pour composer avec ses } \\
\text { émotions et pour résoudre } \\
\text { les conflits }\end{array}$ & 14 & 25,9 & 5 & 55,5 & 8 & 40,0 & 27 & 32,5 \\
\hline $\begin{array}{l}\text { Font en sorte que la } \\
\text { sécurité de l'enfant est } \\
\text { mieux assurée }\end{array}$ & 16 & 29,6 & 4 & 44,4 & 6 & 30,0 & 26 & 31,3 \\
\hline $\begin{array}{l}\text { Favorisent le } \\
\text { développement d'une } \\
\text { meilleure connaissance du } \\
\text { phénomène de la violence } \\
\text { conjugale et familiale }\end{array}$ & 12 & 22,2 & -- & -- & 9 & 45,0 & 21 & 25,3 \\
\hline $\begin{array}{l}\text { Permettent d'améliorer la } \\
\text { relation mère-enfant }\end{array}$ & 7 & 12,9 & 2 & 22,2 & 2 & 10,0 & 11 & 13,2 \\
\hline $\begin{array}{l}\text { Permettent de développer } \\
\text { l'estime de soi de l'enfant }\end{array}$ & 9 & 16,6 & 1 & 11,1 & 1 & 5,0 & 11 & 13,2 \\
\hline $\begin{array}{l}\text { Permettent d'enrichir le } \\
\text { réseau social }\end{array}$ & 5 & 9,2 & -- & -- & 1 & 5,0 & 6 & 7,2 \\
\hline Aident à gérer la crise & 2 & 3,7 & -- & -- & -- & -- & 2 & 2,4 \\
\hline Autres & 8 & 14,8 & -- & -- & -- & -- & 8 & 9,6 \\
\hline
\end{tabular}

* Les pourcentages sont calculés en fonction du nombre de répondantes; leur total peut être supérieur à 100 car les catégories ne sont pas exclusives. 
D'une façon générale, cinq principaux résultats sont attribués aux interventions réalisées auprès des enfants et des adolescents exposés à la violence conjugale. Selon les répondantes, ces interventions : 1) offrent un cadre qui favorise l'expression des émotions suscitées par l'expérience de la violence conjugale, 2) contribuent à réduire les impacts négatifs que cette expérience a sur les enfants et les adolescents, 3) conduisent à l'acquisition de stratégies plus acceptables pour composer avec les émotions négatives et résoudre les conflits, 4) font en sorte que la sécurité de l'enfant est mieux assurée et 5) favorisent le développement d'une meilleure connaissance du phénomène de la violence conjugale et familiale.

\section{TYPOLOGIE DES INTERVENTIONS INDIVIDUELLES}

Les données sur les pratiques d'intervention basées sur l'aide individuelle font ressortir un large éventail de préoccupations et de stratégies dans l'action quotidienne des intervenantes. Bien qu'il soit possible de discerner certaines différences entre les pratiques mises en place dans les différents types d'établissements, il existe également au sein même de chaque type d'établissement une grande diversité dans les façons de faire. Pour dépasser cette hétérogénéité et tenter de regrouper les pratiques autour de traits communs, une analyse de regroupements hiérarchiques (cluster analysis) a été effectuée en introduisant les variables suivantes: le type d'établissement, l'assise théorique principale, l'importance accordée à chaque type d'objectif, la durée et la fréquence des interventions, les moyens utilisés, les thèmes abordés, la place faite aux parents et le recours à une relance. Cette analyse a conduit à diviser les 130 descriptions de pratique (sur 151), pour lesquelles toutes les variables contenaient une donnée valide, en trois groupes formés respectivement de 67, 51 et 12 descriptions, chacun correspondant à un type de pratique.

Dans un deuxième temps, afin de déterminer les éléments qui contribuaient à distinguer ces trois groupes, une analyse discriminante a été effectuée à partir des variables utilisées pour l'analyse de regroupements hiérarchiques. Sept variables ont été retenues. Trois de ces variables sont plus fortement corrélées au premier facteur: la principale assise théorique, le type d'établissement et 
le fait de faire une relance. Ce premier facteur nous semble correspondre principalement aux fondements de l'action. Les quatre autres variables sont associées au facteur 2 : elles font référence à la durée des entrevues, à la fréquence des interventions, aux thèmes abordés et aux moyens utilisés. Ce second facteur traduit principalement les modalités d'action. Le tableau 7 indique de façon plus détaillée en quoi ces trois types de pratiques se distinguent. Les types de pratiques qui émergent se présentent ainsi :

Type $1(n=67)$ : les pratiques féministes formelles. Ce type de pratique présente le profil suivant: l'assise théorique principale est l'approche féministe, les entrevues sont plutôt longues (moy. $=60 \mathrm{~min}$ ) et réalisées à un rythme régulier, les interventions s'appuient sur les échanges verbaux comme moyen d'intervention et une relance est souvent réalisée. Ce type de pratique se retrouve dans les trois types d'établissements.

Type $2(n=12)$ : les pratiques féministes ponctuelles. Dans ce type de pratique, qui prend également assise sur l'approche féministe, la relance est répandue, les activités à caractère ludique occupent une place importance dans les moyens d'action et le thème de la compétence des enfants est souvent abordé. Par ailleurs, les interventions sont de plus courte durée et souvent ponctuelles, c'est-àdire réalisées en fonction des besoins immédiats des enfants. Ce type de pratique, qui se retrouve presque exclusivement en maison d'hébergement, est totalement absent dans les centres jeunesse.

Type $3(n=51)$ : les pratiques éclectiques/systémiques. Ce type de pratique regroupe des interventions dont l'assise théorique principale est l'approche systémique, qui prennent forme dans des entrevues d'environ 50 minutes et qui sont réalisées à un rythme régulier. Les échanges verbaux sont au cœur des moyens d'action privilégiés, alors que les activités ludiques sont relativement peu utilisées. II en est de même de la relance, qui s'y présente comme une pratique plus marginale. Ce type de pratique se retrouve principalement en CLSC, mais il est également présent en centres jeunesse et en maisons d'hébergement. 
Même s'ils traduisent diverses tendances dans les façons de faire, ces trois profils présentent plusieurs traits communs et, en ce sens, ils indiquent que les pratiques mises en place dans les milieux de pratique ne sont pas totalement différentes, bien qu'elles s'inscrivent dans des contextes différents.

\section{Tableau 7 \\ Comparaison entre les trois groupes de profils de pratique sur les variables discriminantes}

\begin{tabular}{|c|c|c|c|c|c|c|}
\hline Variables & $\begin{array}{l}\text { Catégorie } \\
\text { de réponse }\end{array}$ & $\begin{array}{c}\text { Groupe } 1 \\
(n=67)\end{array}$ & $\begin{array}{c}\text { Groupe 2 } \\
(n=12)\end{array}$ & $\begin{array}{c}\text { Groupe } 3 \\
(n=51)\end{array}$ & $X^{2}$ ou $F$ & $\mathbf{P}$ \\
\hline \multicolumn{7}{|l|}{$\begin{array}{l}\text { Principale assise } \\
\text { théorique }^{\mathrm{a}}\end{array}$} \\
\hline - approche féministe & $\mathrm{i}$ & $76,1 \%$ & $58,3 \%$ & --- & 68,91 & $<0,001$ \\
\hline $\begin{array}{l}\text { - approche psycho- } \\
\text { éducationnelle }\end{array}$ & & $16,4 \%$ & $8,3 \%$ & --- & 9,33 & 0,009 \\
\hline - approche éclectique & & $1,5 \%$ & --- & $41,2 \%$ & 35,13 & $<0,001$ \\
\hline - approche systémique & & --- & --- & $25,5 \%$ & 22,37 & $<0,001$ \\
\hline \multicolumn{7}{|l|}{ Type d'établissement ${ }^{a}$} \\
\hline - CLSC & & $50,7 \%$ & $16,3 \%$ & $86,3 \%$ & 26,29 & $<0,001$ \\
\hline - Centre jeunesse & & $11,9 \%$ & --- & $5,9 \%$ & 1,92 & 0,382 \\
\hline - Maison d'hébergement & & $37,3 \%$ & $83,3 \%$ & $7,8 \%$ & 32,54 & $<0,001$ \\
\hline Recours à une relance $^{a}$ & & $55,2 \%$ & $66,7 \%$ & $27,5 \%$ & 11,43 & 0,003 \\
\hline \multirow[t]{3}{*}{$\begin{array}{l}\text { Fréquence des } \\
\text { rencontres }\end{array}$} & $\begin{array}{l}\text { Bihebdo- } \\
\text { madaire }\end{array}$ & $55,2 \%$ & $41,7 \%$ & $47,6 \%$ & \multirow[t]{3}{*}{12,03} & \multirow[t]{3}{*}{0,017} \\
\hline & $\begin{array}{l}\text { Bimensuelle } \\
\text { ou mensuelle }\end{array}$ & $34,3 \%$ & $8,3 \%$ & $33,3 \%$ & & \\
\hline & Au besoin & $10,5 \%$ & $50,0 \%$ & $19,6 \%$ & & \\
\hline Durée des entrevues $^{b}$ & Moyenne & $60 \pm 22$ & $22 \pm 8$ & $50 \pm 12$ & 16,93 & $<0,001$ \\
\hline \multicolumn{7}{|l|}{ Thèmes abordés $^{b}$} \\
\hline $\begin{array}{l}\text { - Compétences des } \\
\text { enfants }\end{array}$ & & $16,4 \%$ & $50,0 \%$ & $31,4 \%$ & 7,65 & 0,022 \\
\hline \multicolumn{7}{|l|}{ Moyens d'intervention $^{\mathrm{b}}$} \\
\hline - Échanges verbaux & & $74,6 \%$ & $50,0 \%$ & $86,3 \%$ & 7,61 & 0,022 \\
\hline - Activités ludiques & & $35,8 \%$ & $66,0 \%$ & $27,5 \%$ & 6,55 & 0,038 \\
\hline
\end{tabular}

${ }^{\mathrm{a}}$ Variables associées au facteur 1.

${ }^{\mathrm{b}}$ Variables associées au facteur 2. 


\section{LES PRATIQUES SPONTANÉES}

La réalisation de cette recherche a servi à identifier des interventions qui s'adressent généralement à un enfant à la fois, mais qui sont réalisées en présence d'autres enfants et parfois aussi en présence d'adultes. II s'agit d'actions qui se situent à mi-chemin entre l'intervention individuelle et l'intervention de groupe. En outre, elles sont généralement spontanées dans la mesure où elles sont provoquées par des événements imprévus. Ce type d'interventions a été recensé dans les maisons d'hébergement pour femmes victimes de violence conjugale; le portrait qui en est tracé s'appuie sur des données recueillies auprès de 62 intervenantes travaillant dans ces organismes. En raison du caractère informel et particulier de ces interventions, il s'est révélé très difficile d'en saisir les contours avec précision. Le portrait présenté ici demeure donc très fragmentaire et ne prétend nullement rendre compte de toute la richesse et de toute la complexité des actions qui sont posées au quotidien dans les maisons d'hébergement à l'endroit des enfants et des adolescents. Toutefois, comme ces pratiques sont peu connues en dehors des maisons d'hébergement, l'information rapportée dans ce texte marque un premier pas vers une meilleure connaissance de la nature de ces actions.

Un premier élément pris en considération touche le contexte dans lequel prennent place les actions spontanées. Selon la majorité des répondantes $(82,2 \%)$, l'arrivée à la maison d'hébergement constitue un moment clé pour la réalisation de ces interventions. Même si l'accueil est une étape bien structurée en maison d'hébergement, les intervenantes doivent souvent faire, auprès des enfants, des actions spontanées. De telles actions prennent également place à des moments de la journée qui marquent la routine quotidienne: l'heure des repas, le moment des devoirs, l'heure du coucher, les moments de détente et de loisirs. Enfin, près de la moitié des répondantes indiquent que ce type d'interventions se situe lors de situations critiques: conflits, comportements agressifs, transgression des règles. 


\section{Tableau 8}

Situations où prennent place les actions spontanées*

\begin{tabular}{|l|c|c|}
\hline \multicolumn{1}{|c|}{ Situations } & $\mathbf{N}=\mathbf{6 2}$ & $\mathbf{\%}$ \\
\hline À l'arrivée dans la maison d'hébergement & 51 & 82,2 \\
\hline À différents moments de la journée & 41 & 66,1 \\
\hline $\begin{array}{l}\text { Dans des situations critiques : conflits, comportements } \\
\text { violents ou agressifs, règles non respectées }\end{array}$ & 30 & 48,3 \\
\hline $\begin{array}{l}\text { À la suite de la demande de la mère ou de sa non- } \\
\text { disponibilité }\end{array}$ & 9 & 14,5 \\
\hline Pour des raisons liées à l'enfant (tristesse, colère, etc.) & 11 & 7,7 \\
\hline
\end{tabular}

* Les pourcentages sont calculés en fonction du nombre de répondantes; leur total est supérieur à 100, car les catégories ne sont pas exclusives.

En ce qui regarde la nature des interventions qui sont faites (tableau 9), deux types d'actions ressortent plus particulièrement, puisqu'elles sont rapportées par plus de la moitié des répondantes: 1) aider à la gestion des conflits de façon pacifique et 2) favoriser l'expression des émotions. Par ailleurs, près de $20 \%$ des répondantes rapportent réaliser des interventions orientées vers le respect des personnes et des règles de la maison ou axées sur la restauration de l'estime de soi des enfants. D'autres interventions portent sur la relation mère-enfant, sur les habiletés parentales de la mère, sur les compétences sociales de l'enfant ou encore sur son bien-être (relaxation). 


\section{Tableau 9}

\section{Les interventions qui sont faites dans le cadre des actions spontanées*}

\begin{tabular}{|l|c|c|}
\hline \multicolumn{1}{|c|}{ Nature des interventions } & $\mathbf{N}=\mathbf{6 2}$ & $\%$ \\
\hline $\begin{array}{l}\text { Aider à gérer des conflits sans violence, de façon } \\
\text { pacifique, à développer de bons comportements, de la } \\
\text { coopération, de bonnes attitudes (en sensibilisant, } \\
\text { expliquant, aidant, donnant des moyens, etc.) }\end{array}$ & 31 & 50,0 \\
\hline Faciliter l'expression des émotions, du vécu & 31 & 50,0 \\
\hline $\begin{array}{l}\text { Faire respecter les personnes et les règles (en le } \\
\text { demandant, en donnant des indications, en appliquant } \\
\text { des méthodes de discipline) }\end{array}$ & 12 & 19,3 \\
\hline $\begin{array}{l}\text { Travailler l'estime de soi, la valorisation et la confiance } \\
\text { en soi, l'affirmation de soi }\end{array}$ & 12 & 19,3 \\
\hline $\begin{array}{l}\text { Agir sur la relation mère-enfant : rassurer mère et } \\
\text { enfant, outiller la mère, etc. }\end{array}$ & 10 & 16,1 \\
\hline $\begin{array}{l}\text { Soutenir la mère dans son rôle parental, la sensibiliser } \\
\text { aux rapports de force }\end{array}$ & 9 & 16,1 \\
\hline $\begin{array}{l}\text { Définir des besoins et/ou diriger vers les bonnes } \\
\text { ressources }\end{array}$ & 5 & 14,5 \\
\hline Travailler la socialisation & 5 & 8,0 \\
\hline Faire de la relaxation & 5 & 8,0 \\
\hline
\end{tabular}

* Les pourcentages sont calculés sur le nombre de répondantes; leur total est supérieur à 100 car les catégories ne sont pas exclusives.

De l'avis des répondantes, ces actions spontanées ont une portée significative sur la situation des enfants et des mères. Les principaux effets qui leur sont attribués gravitent autour des éléments suivants : favorise la confiance en soi et l'estime de soi, contribue à l'adoption de comportements non violents par l'augmentation des connaissances sur la violence et le développement de mécanismes pacifiques de résolution de conflits, augmente la confiance aux autres, contribue à un plus grand sentiment de sécurité et de protection, facilite l'expression des émotions, apporte du soutien et du réconfort et aide l'enfant à développer une meilleure compréhension de la violence. 
Tableau 10

Ce que les actions spontanées apportent aux enfants*

\begin{tabular}{|l|c|c|}
\hline \multicolumn{1}{|c|}{ Effets attribués } & $\mathbf{N}=\mathbf{6 2}$ & $\%$ \\
\hline $\begin{array}{l}\text { Favorise la confiance en soi, l'estime de soi, le } \\
\text { sentiment d'être important }\end{array}$ & 27 & 43,5 \\
\hline $\begin{array}{l}\text { Sensibilise à la violence, permet le développement } \\
\text { de connaissances, d'habiletés d'expression } \\
\text { et de résolution de conflits }\end{array}$ & 25 & 40,3 \\
\hline $\begin{array}{l}\text { Favorise la confiance aux autres, la création / } \\
\text { consolidation de liens }\end{array}$ & 18 & 29,0 \\
\hline Apporte de la sécurité, de la protection, de la stabilité & 17 & 27,4 \\
\hline $\begin{array}{l}\text { Favorise l'expression des sentiments et des émotions, } \\
\text { le partage avec les autres, brise l'isolement }\end{array}$ & 16 & 25,8 \\
\hline Apporte de l'aide, du soutien, du réconfort & 10 & 16,1 \\
\hline Favorise la compréhension de violence & 9 & 14,5 \\
\hline Apporte de l'amour, de l'affection, du respect & 6 & 9,6 \\
\hline $\begin{array}{l}\text { Permet de donner des modèles égalitaires, non } \\
\text { sexistes }\end{array}$ & 22 & 18 \\
\hline
\end{tabular}

* Les pourcentages sont calculés en fonction du nombre de répondantes; leur total est supérieur à 100 , car les catégories ne sont pas exclusives.

\section{Discussion}

Ce portrait global des pratiques d'intervention fait d'abord ressortir que les pratiques basées sur l'aide individuelle présentent plusieurs traits communs. Ainsi, les objectifs poursuivis s'articulent autour d'éléments comme : favoriser l'expression des émotions, atténuer les impacts négatifs de l'exposition à la violence, prévenir la violence dans les relations ultérieures et contribuer à l'apprentissage de scénarios de protection. En ce qui a trait aux thèmes abordés, le phénomène de la violence, la gestion de la colère, l'expression des émotions liées à l'expérience de la violence sont souvent privilégiés. Mais ces pratiques se distinguent également sur des aspects tels que l'assise théorique, la fréquence et la durée des entrevues, de même que les moyens d'intervention privilégiés. Par ailleurs, la portée de ces actions apparaît difficile à mesurer, d'une part, parce que plusieurs objectifs font référence au processus d'intervention plutôt qu'aux changements visés chez les enfants et, d'autre part, parce que les 
objectifs de résultats sont généralement formulés dans des termes généraux et font, pour plusieurs, référence à des changements à moyen et à long terme. II n'est donc pas étonnant que les procédures d'évaluation utilisées soient informelles et qu'elles reposent sur l'appréciation des intervenants, des enfants et des parents.

Les données font également ressortir que les pratiques spontanées sont répandues, même si elles sont peu connues à l'extérieur du cadre des maisons d'hébergement. Leur caractère informel fait cependant en sorte qu'il est très difficile d'en donner une description précise. Pour en arriver à cerner avec précision la nature et la portée de ces interventions, il faudrait les documenter de façon systématique, au moment même où elles se produisent.

La réalité des enfants qui vivent dans un contexte familial marqué par la violence entre les conjoints est un phénomène de mieux en mieux connu, du moins dans ses conséquences à court terme. Évidemment, la prévalence du problème demeure difficile à estimer, de même que son impact à plus long terme sur le fonctionnement social et sur les relations intimes; toutefois, les recherches récentes confirment que les enfants exposés à la violence conjugale connaissent des problèmes sur le plan de leur développement émotionnel et comportemental (Kolbo et collab., 1996). II est donc nécessaire de développer des actions pour venir en aide aux enfants dont le quotidien est truffé d'injures, de menaces, de coups, de blessures, de pleurs, de désespoir et de mensonges.

Les premières actions en ce qui concerne la violence conjugale ont été orientées vers les femmes; d'une part, parce qu'elles en étaient les premières victimes, d'autre part, parce qu'elles étaient considérées comme les personnes les mieux placées pour venir en aide à leurs enfants. Les observations sur le désarroi des femmes lorsqu'elles traversent une période de crise et sur les réactions des enfants exposés à la violence conjugale ont fait prendre conscience de la nécessité d'intervenir directement auprès des enfants et des adolescents. II faut cependant garder à l'esprit que les actions auprès des enfants auxquelles les mères ne sont pas parties prenantes risquent d'avoir une portée beaucoup plus restreinte, d'où l'importance de maintenir et d'intensifier les efforts pour associer à l'intervention les personnes 
qui sont proches de l'enfant. D'où la nécessité, également, de promouvoir des interventions à d'autres niveaux pour dénoncer la violence, changer les valeurs, modifier les rapports sociaux entre les hommes et les femmes et faire la promotion de conduites égalitaires et pacifiques. Pour contrer la violence et ses effets, il est essentiel d'agir non seulement sur les personnes, mais aussi sur leur environnement social et politique, comme le préconise la perspective féministe.

Tout en poursuivant les actions actuelles, il est essentiel de réfléchir aux nouvelles avenues qui pourraient être avantageusement empruntées pour, à plus long terme, éliminer la violence et, à plus court terme, en atténuer les effets. Actuellement, un débat important est en cours sur la nécessité d'encadrer à l'intérieur de paramètres légaux l'intervention auprès des enfants exposés à la violence conjugale. Par la position qu'ils occupent, les intervenantes et les intervenants sont bien placés pour s'interroger sur les forces et les limites des actions actuelles et pour collaborer au développement de nouvelles voies d'action adaptées aux besoins des enfants et des adolescents exposés à la violence conjugale.

Daniel TURCOTTE Professeur École de service social, Université Laval

Ginette BEAUDOIN Professionnelle de recherche Cri-Viff, Université Laval

Ann PÂQUET-DEEHY Professeure École de service social, Université de Montréal 


\section{Références bibliographiques}

AlEssi, J.J. et K. HEARN (1984). "Group Treatment of Children in Shelters for Battered Women », dans A.R. Roberts (dir.), Battered Women and Their Families (p. 49-61), New York, Springer.

ARRoYo, W. et S. ETH (1995). "Assessment Following ViolenceWitnessing Trauma », dans E. Peled, P.G. Jaffe et J. Edleson (dir.), Ending the Cycle of Violence, Thousand Oaks, Sage.

Beaudoin, G., I. Côté, R. Delisle, M.-C. Gaboury, N. Guénette et M. LESSARD (1998). "L'intervention de groupe au service des enfants exposés à la violence conjugale », Intervention, $\mathrm{n}^{\circ} 107, \mathrm{p}$. 19-32.

BELSKI, J. (1984). «Two Waves of Day Care Research : Developmental Effects and Conditions of Quality », dans R. Ainslie (dir.), The Child and Day Care Setting, New York, Praeger.

Blanchet, L., M.-C. Laurendeau, D. Paul et J.-F. Saucier (1993). La prévention et la promotion en santé mentale, Boucherville, Gaëtan Morin.

Burman, S. et P. Allen-Meares (1994). « Neglected Victims of Murder: Children's Witness to Parental Homicide », Social Work, vol. 39, no 1, p. 28-34.

CARLSON, B.E. (1990). "Adolescent Observers of Marital Violence", Journal of Family Violence, vol. 5, $n^{\circ}$ 4, p. 285-299.

CHÉNARD, L. (1994). « ... et les enfants », dans Maryse Rinfret-Raynor et Solange Cantin (dir.), Violence conjugale: Recherches sur la violence faite aux femmes en milieu conjugal, Boucherville, Gaëtan Morin Éditeur, p. 113-129.

ChÉNARD, L., H. CADRIN et J. LoISELLE (1990). État de santé des femmes et des enfants victimes de violence conjugale. Rapport de recherche, Département de santé communautaire, Centre hospitalier régional de Rimouski.

DAVIS, D. (1991). "Intervention with Male Toddlers Who Have Witnessed Parental Violence ", The Journal of Contemporary Human Services, $n^{\circ} 11$, p. 515-524. 
Fantuzzo, J.W., L.M. DePaola, L. Lambert, T. Martino, G. Anderson et S. SUTTON (1991). "Effects of Interparental Violence on the Psychological Adjustment and Competencies of Young Children », Journal of Consulting and Clinical Psychology, vol. 59, $n^{\circ} 2$, p. 258265.

FANTUZZO, J.W. et C.U. LINDQUIST (1989). «The Effects of Observing Conjugal Violence on Children: A Review and Analyses of Research Methodology ", Journal of Family Violence, vol. 4, no 1, p. 77-93.

Forsstrom-Cohen, B. et A. Rosenbaum (1985). "The Effects of Parental Marital Violence on Young Adults: An Exploratory Investigation », Journal of Marriage and Family, vol. 47, p. 467-471.

GABOURY, M.-C. et N. GuÉNETTE (1999). « La parole est aux enfants », Le Bulletin, Regroupement des centres de la petite enfance de la Montérégie, vol. $20, \mathrm{n}^{\circ}$ 2, p. 18-21.

GibSON, J.W. et L. GutieRREZ (1991). " A Service Program for SafeHome Children », Families in Society, vol. 72, p. 554-562.

GLODICH, A. (1998). «Traumatic Exposure to Violence: A Comprehensive Review of the Child and Adolescents Literature ", Smith College Studies in Social Work, vol. 68, p. 321-345.

GRUNSZKI, R., J. BRINK et J. EDLESON (1988). " Support and Education Groups for Children of Battered Women », Child Welfare, vol. 67, $n^{\circ} 5$, p. 431-444.

Guberman, N., J. BRouÉ, J. Lindsay et L. Spector (1993). Le défi de l'égalité : La santé mentale des hommes et des femmes. Comité de la santé mentale du Québec, Boucherville, Gaëtan Morin.

Hennings, K., H. Leintenderg, P. Coffey, T. TuRner et R.T. BennetT (1996). « Long-Term Psychological and Social Impact of Witnessing Physical Conflict Between Parents », Journal of Interpersonal Violence, vol. 11, $\mathrm{n}^{\circ}$ 1, p. 35-51.

Holden, G.W. (1998). «Introduction: The Development of Research into Another Consequence of Family Violence », dans G.W. Holden, R. Geffner et E.N. Jouriles (dir.), Children Exposed to Marital Violence : Theory, Research and Applied Issues, p. 1-18.

HUGHES, H.M. (1982). "Brief Intervention with Children In a Battered Women's Shelter: A Model Preventive Program », Family Relations, vol. 31 , no 4 , p. 495-502. 
HUGHES, H.M. (1988). "Psychological and Behavioral Correlates of Family Violence in Child Witnesses and Victims ", American Journal of Orthopsychiatry, vol. 18, p. 77-90.

HugHES, H.M. (1997). "Research Concerning Children of Battered Women: Clinical Implications », Journal of Aggression, Maltreatment and Trauma, vol. 1, p. 221-239.

HUGHES, H.M. et S.J. BARAD (1983). "Psychological Functioning of Children in a Batterer Women's Shelter: A Preliminary Investigation », American Journal of Orthopsychiatry, vol. 18, p. 77-90.

HugheS, H.M., D. PARKINSON et M. VARGO (1989). "Witnessing Spouse Abuse and Experiencing Physical Abuse : A "Double Whammy" ?», Journal of Family Violence, vol. 5, n 4, p. 285-299.

JAFFE, P.G., S.K. WILSON et D.A. WolfE (1986). « Promoting Changes in Attitudes and Understanding of Conflict Resolution Among Child Witnesses of Family Violence », Canadian Journal of Behavioral Science, vol. $18, n^{\circ} 4$, p. 356-380.

JAFFe, P.G., D.A. Wolfe, S.K. Wilson et L. ZAK (1985). "Problèmes critiques mis en lumière par une étude sur l'adaptation des enfants témoins de violence dans la famille ", Santé mentale au Canada, vol. 33, n 4, p. 15-19.

JAFFE, P.G., D.A. WOLFE, S.K. WILSON et L. ZAK (1986). "Similarities in Behavioral and Social Maladjustment Among Child Victims and Witnesses to Family Violence ", American Journal of Orthopsychiatry, vol. 56, $\mathrm{n}^{\circ} 1$, p. 142-146.

JAFFE, P.G., D.A. Wolfe et S.K. WILSON (1990). Children of Battered Women, vol. 21, Londres, Sage, 132 p.

JAFFe, P.G., M. Sudermann et D. Reitzel (1992). «Child Witnesses of Marital Violence ", dans R.T. Ammerman et M. Hersen (dir.), Assessment of Family Violence: A Clinical and Legal Sourcebook (p. 313-331), New York, John Wiley \& Sons, Inc.

JoHnson, H. (1996). Dangerous Domains: Violence Against Women in Canada, Toronto, Nelson Canada, 252 p.

Johnson, R.J. et M. MONTGOMERY (1990). «Children at Multiple Risk : Treatment and Prevention», dans R.T. Potter-Efron et P.S. PotterEfron (dir.), Aggression, Family Violence and Chemical Dependency (p. 145-163), New York, Haworth Press. 
Jouriles, E.N., R. McDonald, N. Stephens, W. Norwood, S. Collazos et H.S. WARE (1998). "Breaking the Cycle of Violence: Helping Families Departing From Battered Women's Shelters », dans G.W. Holden, R. Geffner et E.N. Jouriles (dir.), Children Exposed to Marital Violence: Theory, Research and Applied Issues, Washington, American Psychological Association, p. 337-370.

KéRouAK, S., M.E. TAggaRT et J. Lescop (1986). Portrait de la santé de femmes violentées et de leurs enfants, Faculté des sciences infirmières, Université de Montréal.

KILPATRICK, K.L. et L.M. WILLIAMS (1997). "Post-traumatic Stress Disorder in Child Witnesses to Domestic Violence », American Journal of Orthopsychiatry, vol. 67, p. 639-644.

Kolbo, J.R., E.H. Blakely et D. Engleman (1996). «Children Who Witness Domestic Violence: A Review of Empirical Literature», Journal of Interpersonal Violence, vol. 11, $\mathrm{n}^{\circ}$ 2, p. 281-293.

LANCUP, S. et L. VAILLANT (1996). «Contrer la violence faite aux enfants dans la famille : Un choix de société », dans J. Coiteux, P. Campeau, M. Clarkson et M.-M. Cousineau (dir.), Question d'équité : L'aide aux victimes d'actes criminels. Association québécoise PlaidoyerVictimes, p. 275-299.

Marengère, D., L. Champagne et P.-P. Poirier (1998). «Groupe pour enfants exposés à la violence conjugale », Intervention, $\mathrm{n}^{\circ} 107, \mathrm{p}$. 62-67.

MCKAY, M.M. (1994). "The Link Between Domestic Violence and Child Abuse : Assessment and Treatment Considerations », Child Welfare, vol. 73, n 1, p. 29-39.

MCLeOD, L. (1987). Pour de vraies amours... Prévenir la violence conjugale, Ottawa, Conseil consultatif canadien sur la situation de la femme.

MERCIER, C. (1985). "L'évaluation des ressources alternatives. À la recherche de modèles alternatifs en évaluation", Revue canadienne de santé communautaire, vol. 4, p. 57-72.

MoRRIS, L. et C. FITZ-GIBBON (1978). Evaluator's Handbook, Beverly Hills, Sage.

MSSS (1991). Un Québec fou de ses enfants. Rapport du groupe de travail pour les jeunes, Gouvernement du Québec, $179 \mathrm{p}$.

PÂquet-Deehy, A., J. Proulx, C. Hamel avec la collaboration de H. CHOUÉRI (1997). L'évaluation d'une intervention de groupe auprès d'enfants et d'adolescents exposés à la violence conjugale 
aux centres jeunesse de la famille et de la famille Batshaw. Les Centres de la jeunesse et de la famille Batshaw, $275 \mathrm{p}$.

Peled, E. et J.L. EdLeson (1992). « Multiple Perspectives on Groupwork with Children of Battered Women ", Violence and Victims, vol. 7, $\mathrm{n}^{\circ} 4$, p. 327-346.

Peled, E. (1993). "Children Who Witness Women Battering: Construction of Social Problem », Children and Youth Services Review, vol. 15, p. 43-52.

PELED, E. et D. DAVIS (1995). Groupwork with Children of Battered Women : A Practitioner's Manual, Londres, Sage, 225 p.

Peled, E. et J.L. Edleson (1995). "Process and Outcome in Small Groups for Children of Battered Women », dans E. Peled, P.G. Jaffe et J.L. Edleson (dir.), Ending the Cycle of Violence: Community Responses to Children of Battered Women, Thousand Oaks, CA, Sage Publications.

Peled, E. (1997). "Intervention with Children of Battered Women : a Review of Current Literature ", Children and Youth Services Review, vol. 19, n 4, p. 277-299.

PetCheRs, M.K. (1995). Child Maltreatment Among Children in Battered Mother's Households. Communication présentée à la $4^{\mathrm{e}}$ Conférence internationale sur la recherche familiale, Durham, New Hampshire, États-Unis.

PoweLL, D. (1988). « Client Characteristics and the Design of CommunityBase Intervention Programs ", dans A. Pence (dir.), Ecological Research with Chidren and Families, New York, Columbia Univ., Teachers College Press.

Rinfret-Raynor, M., A. PÂquet-Deehy, G. Larouche et S. CANTIN (1992). Intervenir auprès des femmes violentées: Évaluation de l'efficacité d'un modèle féministe, Montréal, Éditions Saint-Martin, $80 \mathrm{p}$.

Rosenbaum, A. et K.D. O'Leary (1981). "Children: The Unintended Victims of Marital Violence », American Journal of Orthopsychiatry, vol. 51, p. 692-699.

RoY, M. (1982). The Abusive Partner: An Analysis of Domestic Battering, New York, V.N.R.

RUdo, Z.H., D.S. PoWELL et G. DUNLAP (1998). «The Effects of Violence in the Home on Children's Emotional, Behavioral, and Social Functioning : A Review of the Literature ", Journal of Emotional and Behavioral Disorders, vol. 6, p. 94-113. 
SILVERN, L., J. KARYL et T.Y. LANDIS (1995). «Individual Psychotherapy for the Traumatized Children of Abused Women », dans E. Peled, P.G. Jaffe et J.L. Edleson (dir.), Ending the Cycle of Violence: Community Responses to Children of Battered Women, Thousand Oaks, CA, Sage.

SONKIN, D.J., D. MARTIN et L.E. WALKER (1985). The Male Batterers : A Treatment Approach, New York, Springer Publishing Company, 256 p.

Straus, M.A., R.J. Gelles et S.K. Steinmetz (1980). Behind Closed Doors : Violence in the American Family, Doubleday Press, Garden City.

TURCOTTE, D. et J.R. FOURNIER (1994). Les pratiques actuelles en service social des groupes. Nature et contraintes, École de service social, Université Laval.

WeISS, H. (1988). «Family Support and Education Programs : Working Through Ecological Theories of Human Development », dans $\mathrm{H}$. Weiss et F. Jacobs (dir.), Evaluating Family Programs, New York, Aldine de Gruyter.

WESTRA, B.L. et H.P. MARTIN (1981). "Children of Battered Women », Maternal Child Nursing Journal, vol. 10, p. 41-51.

Whitebook, M., C. Howes et P. PHILIPPS (1989). Who Cares? Child Care Teachers and the Quality of Care in America : Final Report National Child Care Staffing Study, Oakland, Child Care Employee Project.

WOLAK, J. et D. FINKELHOR (1998). «Children Exposed to Partner Violence ", dans Partner Violence : A Comprehensive Review of 20 Years of Research, J.L. Jasinski et L.M. Williams (dir.), Thousand Oaks, Sage, p. 111.

WolfE, D.A., P. JAFFE, S.K. WILSON et L. ZAK (1985). "Children of Battered Women: The Relationship of Child Behavior to Family Violence and Maternel Stress », Journal of Consulting and Clinical Psychology, vol. 53, n ${ }^{\circ}$ 5, p. 657-665.

WolFE, D.A. (1999). Comprendre et traiter les enfants témoins de violence domestique. Séminaire de formation tenu à Ottawa les 26 et 27 avril 1999. 